\title{
SELFADJOINT REPRESENTATIONS OF POLYNOMIAL ALGEBRAS
}

BY

\author{
ATSUSHI INOUE AND KUNIMICHI TAKESUE
}

\begin{abstract}
In this paper we will investigate the selfadjointness of unbounded *-representations of the polynomial algebra. In particular, it is shown that the notion of selfadjoint representation is equivalent to that of standard representation in the case of the polynomial algebra generated by one hermitian element. Although the notion of standardness implies that of selfadjointness, the converse is not true in general. Therefore, we consider under what conditions $a^{*}$-representation is standard.
\end{abstract}

Introduction. In [8] Powers investigated unbounded *-representations of a *-algebra and pointed out that there exists a pathological relation between subrepresentations and commutants which does not occur in the bounded case. Over ten years has passed since he introduced the notion of selfadjoint representation in order to take out such a phenomenon. During this time many mathematicians have studied unbounded *-representations $[1,4,6,8-10]$. The notion of selfadjointness has been indispensable in order to make a detailed study. However, since the great difficulty lies in the judgment of selfadjointness, it seems that sufficient study has not been done. From this viewpoint, we will study conditions under which a *-representation is selfadjoint. Even on the *-representation of the polynomial algebra generated by finite commuting hermitian elements, we have obtained interesting results, which we state here.

1. Preliminaries. We introduce some notions which are used in this paper, for example, $O_{p}^{*}$-algebra, representation, etc.

Let $\mathscr{D}$ be a dense subspace of a Hilbert space $\mathfrak{h}$. By $\mathcal{L}(\mathscr{D})$ we denote the set of all linear operators of $\mathscr{D}$ into $\mathscr{D}$. Then $\mathscr{L}(\mathscr{D})$ is an algebra under the usual operations. By $\mathcal{L}^{+}(\mathscr{Q})$ we denote the set of all linear operators $A$ in $\mathcal{L}(\mathscr{D})$ such that $\mathscr{D}\left(A^{*}\right) \supset \mathscr{D}$ and the restriction $A^{+}$of $A^{*}$ to $\mathscr{D}$ is contained in $\mathcal{L}(\mathscr{D})$, where $A^{*}$ is the adjoint of $A$. Then $\mathfrak{L}^{+}(\mathscr{D})$ becomes a ${ }^{*}$-algebra under the usual operations and the involution $A \rightarrow A^{+}$. For a subalgebra $\Re$ of $\mathcal{L}(\mathscr{D}),(\Re, \mathscr{D})$ is said to be an $O_{p}$-algebra, and for a *-subalgebra $\mathfrak{T}$ of $\mathfrak{L}^{+}(\mathscr{D}),(\mathscr{T}, \mathscr{D})$ is said to be an $O_{p}^{*}$-algebra. Let $(\mathfrak{T}, \mathscr{D})$ be an $O_{p}$-algebra. We define a seminorm \|\|$_{A}$ on $\mathscr{D}$ for $A \in \mathcal{N}_{I}$ by $\|\xi\|_{A}=\|A \xi\|$, where $\Re_{I}$ is the $O_{p}$-algebra obtained by adjoining an identity operator to $\Re$. The induced topology $t_{\mathfrak{T}}$ on $\mathscr{D}$ is the locally convex topology generated by the collection of

Received by the editors July 22, 1982.

1980 Mathematics Subject Classification. Primary 47D40, 46K10; Secondary 46L10.

Key words and phrases. $O_{p}^{*}$-algebra, (closed, selfadjoint, standard) representation. 
seminorms $\left\{\|\|_{A} ; A \in \Re_{I}\right\}$. If $\mathscr{D}$ is complete in the induced topology $t_{\mathscr{N}}$, then $\left(\mathscr{T}_{R}, \mathscr{Q}\right)$ is called closed. The closedness of $\Re$ is equivalent to $\mathscr{D}=\cap_{A \in \mathscr{N}} \mathscr{D}(\bar{A})$, where $\mathscr{D}(\bar{X})$ denotes the domain of the closure $\bar{X}$ of a closable operator $X$. Let $(\mathscr{N}, \mathscr{D})$ be an $O_{p}^{*}$-algebra. If $\mathscr{D}_{*}(\mathscr{T}) \equiv \cap_{A \in \Re} \mathscr{D}\left(A^{*}\right)=\mathscr{D}$, then $(\mathscr{N}, \mathscr{D})$ is called

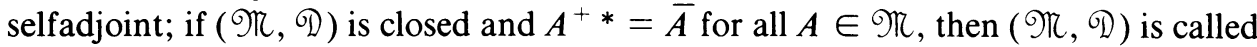
standard. We next define a commutant $\mathscr{T} \mathcal{R}^{\prime}$ of an $O_{p}^{*}$-algebra $(\mathfrak{R}, \mathscr{D})$ as follows:

$$
\mathfrak{N}^{\prime}=\left\{C \in \mathscr{B}(\mathfrak{h}) ;(C A \xi \mid \eta)=\left(C \xi \mid A^{+} \eta\right) \text { for all } A \in \mathfrak{N} \text { and } \xi, \eta \in \mathscr{D}\right\},
$$

where $\mathscr{B}(\mathfrak{h})$ denotes the set of all bounded linear operators on $\mathfrak{h}$. If $C \in \mathfrak{N}^{\prime}$, then $C \mathscr{D} \subset \mathscr{D}_{*}(\mathscr{N})$ and $\left(A^{+}\right)^{*} C \xi=C A \xi$ for all $A \in \mathscr{N}$ and $\xi \in \mathscr{Q}$. The commutant $\Re^{\prime}$ of $\mathfrak{N}$ is not necessarily an algebra; however if $\Re$ is selfadjoint, then $\mathfrak{K}^{\prime}$ is a von Neumann algebra and $\mathscr{N} \mathscr{D}=\mathscr{Q}[8]$.

Let $Q$ be a ${ }^{*}$-algebra. A homomorphism (resp. ${ }^{*}$-homomorphism) $\pi$ of $\mathcal{Q}$ onto an $O_{p}$-algebra (resp. an $O_{p}^{*}$-algebra), on $\mathscr{D}$ is said to be a representation (resp. a *-representation) of $Q$ on $\mathfrak{h}$ with domain $\mathscr{D}(\pi)=\mathscr{Q}$. A representation $\pi$ of $\mathscr{Q}$ is closed if the $O_{p}$-algebra $(\pi(Q), \mathscr{D}(\pi))$ is closed; and a ${ }^{*}$-representation $\pi$ of $Q$ is called selfadjoint (resp. standard) if $(\pi(\mathbb{Q}), \mathscr{D}(\pi))$ is selfadjoint (resp. standard).

Let $\pi, \rho$ be representations of $\mathcal{Q}$ on a Hilbert space $\mathfrak{h}$. If $\mathscr{D}(\rho) \subset \mathscr{D}(\pi)$ and $\rho(x) \xi=\pi(x) \xi$ for all $x \in \mathbb{Q}$ and $\xi \in \mathscr{D}(\rho)$, then we say that $\pi$ is an extension of $\rho$, denoted by $\rho \subset \pi$.

Let $\pi$ be a representation of a ${ }^{*}$-algebra $\mathfrak{Q}$ on a Hilbert space $\mathfrak{h}$. Then we define closed representations of $\mathscr{Q}$ as follows:

$$
\begin{array}{ll}
\mathscr{D}(\tilde{\pi})=\bigcap_{x \in \mathbb{Q}} \mathscr{D}(\overline{\pi(x)}), & \tilde{\pi}(x)=\overline{\pi(x)} / \mathscr{D}(\tilde{\pi}) \\
\mathscr{D}\left(\pi^{*}\right)=\bigcap_{x \in \mathbb{Q}} \mathscr{D}\left(\pi(x)^{*}\right), \quad \pi^{*}(x)=\pi\left(x^{*}\right)^{*} / \mathscr{D}\left(\pi^{*}\right) & \text { for } x \in \mathbb{Q} .
\end{array}
$$

If $\mathscr{D}\left(\pi^{*}\right)$ is dense in $\mathfrak{h}$, then the closed representation $\pi^{* *}$ of $\mathscr{Q}$ is defined by

$$
\mathscr{D}\left(\pi^{* *}\right)=\bigcap_{x \in \mathbb{Q}} \mathscr{D}\left(\pi^{*}(x)^{*}\right), \quad \pi^{* *}(x)=\pi^{*}\left(x^{*}\right)^{*} / \mathscr{D}\left(\pi^{* *}\right) \quad \text { for } x \in \mathbb{Q} .
$$

Then we see that if $\pi$ is $a^{*}$-representation, then $\tilde{\pi}$ and $\pi^{* *}$ are ${ }^{*}$-representations satisfying the condition $\pi \subset \tilde{\pi} \subset \pi^{* *} \subset \pi^{*}$.

2. Selfadjointness of $\mathscr{P}(A)$. We consider the problem mentioned in the Introduction for ${ }^{*}$-representations of the polynomial algebra generated by one hermitian element.

THEOREM 2.1. Let $\mathscr{D}$ be a dense subspace of a Hilbert space $\mathfrak{h}, A$ an hermitian element of $\mathcal{L}^{+}(\mathcal{D})$ and $\mathcal{P}(A)$ the polynomial algebra generated by $A$. Suppose the $O_{p}^{*}$-algebra $(\mathscr{P}(A), \mathscr{D})$ is closed. Then the following statements are equivalent.

(1) $(\mathscr{P}(A), \mathscr{D})$ is standard.

(2) $(\mathscr{P}(A), \mathscr{D})$ is selfadjoint.

(3) $\mathscr{P}(A)^{\prime} \mathscr{D}=\mathscr{Q}$.

(4) $A$ is essentially selfadjoint and $\mathscr{D}=\bigcap_{n=1}^{\infty} \mathscr{D}\left(\bar{A}^{n}\right)$.

(5) $A^{n}$ is essentially selfadjoint for $n=1,2, \ldots$ 
Proof. The implications (1) $\Rightarrow(2) \Rightarrow(3)$ are clear.

(3) $\Rightarrow(4)$ : We first show that $\mathcal{P}(A)^{\prime}$ is an algebra. Let $C_{1}, C_{2}$ be elements of $\mathscr{P}(A)^{\prime}$. Since $\bar{P}(A)^{*} C_{2} \xi=C_{2} P(A) \xi$ for each $\xi \in \mathscr{D}$ and $P(A) \in \mathcal{P}(A)$, and $\mathscr{P}(A)^{\prime} \mathscr{D}=\mathscr{D}$, we have $P(A) C_{2} \xi=C_{2} P(A) \xi$. Therefore, $C_{1} C_{2} P(A) \xi=C_{1} P(A) C_{2} \xi=P(A) C_{1} C_{2} \xi$. Hence, $\mathcal{P}(A)^{\prime}$ is an algebra. By [8, Lemma 3.2], $A$ is essentially selfadjoint. Let $\bar{A}=\int_{-\infty}^{\infty} \lambda d E(\lambda)$ be the spectral resolution and $x$ an element of $\cap_{n=1}^{\infty} \mathscr{D}\left(\bar{A}^{n}\right)$. Then there exists a sequence $\left\{\xi_{n}\right\}$ in $\mathscr{D}$ with $\lim _{n \rightarrow \infty} \xi_{n}=x$. We put $E_{n}=\int_{-n}^{n} d E(\lambda)$. Then $E_{n} \in \mathscr{P}(A)^{\prime}$. Hence, $E_{n} \xi_{k} \in \mathcal{P}(A)^{\prime} \mathscr{D}=\mathscr{D}$. Furthermore, since $\lim _{k \rightarrow \infty} E_{n} \xi_{k}=E_{n} x$ and $\lim _{k \rightarrow \infty} A_{m} E_{n} \xi_{k}=\bar{A}_{m} E_{n} x(n, m=1,2, \ldots)$, and $(\mathscr{P}(A), \mathscr{D})$ is closed, we have $E_{n} x \in$ Q . Since $\lim _{n \rightarrow \infty} E_{n} x=x$ and $\lim _{n \rightarrow \infty} A^{m} E_{n} x=\bar{A}^{m} x$ for $m=1,2, \ldots$, we have $x \in \mathscr{O}$ ).

(4) $\Rightarrow(1)$ : Let $\bar{A}=\int_{-\infty}^{\infty} \lambda d E(\lambda)$ be the spectral resolution of the selfadjoint operator $\bar{A}$ and $E_{n}$ the projection defined by $E_{n}=\int_{-n}^{n} d E(\lambda)(n=1,2, \ldots)$. Let $P(t)$ be a polynomial. Then $P(\bar{A})$ is defined by

$$
P(\bar{A})=\int_{-\infty}^{\infty} P(\lambda) d E(\lambda)
$$

and

$$
\text { domain } \mathscr{D}(P(\bar{A}))=\left\{x \in \mathfrak{h} ; \int_{-\infty}^{\infty}|P(\lambda)|^{2} d\|E(\lambda) x\|^{2}<\infty\right\} \text {. }
$$

It is well known that $P(\bar{A})$ is a closed operator. Clearly we have $P(A) \subset P(\bar{A})$. Let $x$ be an element of $\mathscr{D}(P(\bar{A}))$. Then $E_{n} x \in \mathcal{D}$ for $n=1,2, \ldots$ Since $\lim _{n \rightarrow \infty} \underline{E_{n} x=x}$ and $\lim _{n \rightarrow \infty} P(A) E_{n} x=P(\bar{A}) x$, we have $x \in \mathscr{D} \overline{(P(A))}$. This means that $\frac{E_{n}(A)}{P(A)}$ $P(\bar{A})$. Consequently, we have

$$
\bar{P}(A)^{*}=\overline{\bar{P}(A)}{ }^{*}=\bar{P}(\bar{A})^{*}=P(\bar{A})=\overline{P(A)} .
$$

Therefore, $(\mathscr{P}(A), \mathscr{D})$ is standard.

(1) $\Rightarrow(5)$ : This is trivial.

$(5) \Rightarrow(4)$ : Since $\mathscr{D}$ is closed, we have $\mathscr{D}=\bigcap_{n \equiv 1}^{\infty} \mathscr{D}\left(\bar{A}^{n}\right)$. By the assumption, $\overline{A^{n}}=\overline{A^{n}}$ for $n=1,2, \ldots$ and, hence, $\mathscr{D}=\bigcap_{n=1}^{\infty} \mathscr{D}\left(\overline{A^{n}}\right)$.

LEMMA 2.2. If $A$ is an element of $\mathcal{L}(\mathcal{D})$ and $n$ is a natural number, then

$$
\text { (ㅇ) }\left(A^{* n}\right)=\bigcap_{k=1}^{n} \text { oD }\left(A^{k *}\right) \text { and } A^{* k} \xi=A^{k *} \xi
$$

for $\xi \in \mathcal{O D}\left(A^{* n}\right)$ and $k=1,2, \ldots, n$. In particular, we have

$$
\bigcap_{n=1}^{\infty} \mathscr{D}\left(A^{* n}\right)=\bigcap_{n=1}^{\infty} \mathscr{D}\left(A^{n *}\right) \text {. }
$$

Proof. We prove this by irduction. It is clear for $n=1$. We suppose it holds for $n$. Since $\left(A^{*}\right)^{n+1} \subset\left(A^{n+1}\right)^{*}$, we have

$$
\mathscr{D}\left(\left(A^{*}\right)^{n+1}\right) \subset \bigcap_{k=1}^{n+1} \mathscr{D}\left(A^{k *}\right)
$$

and

$$
\left(A^{n+1}\right)^{*} \xi=\left(A^{*}\right)^{n+1} \xi \quad \text { for } \xi \in \mathscr{D}\left(\left(A^{*}\right)^{n+1}\right)
$$


For each $\xi \in \bigcap_{k=1}^{n+1} \mathscr{D}\left(A^{k *}\right)$ and $\eta \in \mathscr{D}$ we have

$$
\left(A \eta \mid A^{* n} \xi\right)=\left(A \eta \mid A^{n *} \xi\right)=\left(A^{n+1} \eta \mid \xi\right)=\left(\eta \mid\left(A^{n+1}\right) * \xi\right)
$$

which implies that $A^{* n} \xi \in \mathscr{D}\left(A^{*}\right)$ and $A^{*} A^{* n} \xi=\left(A^{*}\right)^{n+1} \xi=\left(A^{n+1}\right)^{*} \xi$.

LEMMA 2.3. If $\pi$ is $a^{*}$-representation of $a^{*}$-algebra $\mathbb{Q}$, then $\pi^{* *}(\mathbb{Q})^{\prime}=\pi(\mathbb{Q})^{\prime}$.

Proof. It is clear that $\pi^{* *}(\mathscr{Q})^{\prime} \subset \pi(\mathbb{Q})^{\prime}$. Let $C$ be an element of $\pi(\mathscr{Q})^{\prime}$. For each $x \in \mathbb{Q}, \xi \in \mathscr{D}(\pi)$ and $\eta \in \mathscr{D}\left(\pi^{* *}\right)$ we have

$$
\begin{aligned}
\left(C \pi^{* *}(x) \eta \mid \xi\right) & =\left(\pi^{* *}(x) \eta \mid C^{*} \xi\right)=\left(\pi^{*}\left(x^{*}\right)^{*} \eta \mid C^{*} \xi\right) \\
& =\left(\eta \mid \pi^{*}\left(x^{*}\right) C^{*} \xi\right) \quad\left(\text { by } C^{*} \xi \in \mathscr{D}\left(\pi^{*}\right)\right) \\
& =\left(\eta \mid C^{*} \pi\left(x^{*}\right) \xi\right) \quad\left(\text { by } C \in \pi(\mathbb{Q})^{\prime}\right) \\
& =\left(C \eta \mid \pi\left(x^{*}\right) \xi\right) .
\end{aligned}
$$

Hence, $C \eta \in \mathscr{D}\left(\pi^{*}\right)$ and $C \pi^{* *}(x) \eta=\pi^{*}(x) C \eta$ for each $x \in \mathbb{Q}$ and $\eta \in \mathscr{Q}\left(\pi^{* *}\right)$, which implies that $C \in \pi^{* *}(\mathbb{Q})^{\prime}$.

THEOREM 2.4. Let $\mathscr{P}\left(x_{0}\right)$ be the free commutative algebra generated by one hermitian element $x_{0}$ and $\pi a{ }^{*}$-representation of $\mathcal{P}\left(x_{0}\right)$ on a Hilbert space. Then $\pi^{*}$ is selfadjoint if and only if $\pi\left(x_{0}\right)$ is essentially selfadjoint.

Proof. Suppose that $\pi^{*}$ is selfadjoint; that is, $\pi^{*}=\pi^{* *}$. Then $\pi^{*}\left(\mathscr{P}\left(x_{0}\right)\right)^{\prime}$ is an algebra. By Lemma $2.3 \pi\left(\mathscr{P}\left(x_{0}\right)\right)^{\prime}$ is an algebra. It therefore follows from [8, Lemma 3.2] that $\pi\left(x_{0}\right)$ is essentially selfadjoint. Conversely, suppose that $\pi\left(x_{0}\right)$ is essentially selfadjoint. By Lemma 2.2 we have

$\mathscr{D}\left(\pi^{*}\right)=\bigcap_{n=1}^{\infty} \mathscr{D}\left(\pi\left(x_{0}^{n}\right)^{*}\right)=\bigcap_{n=1}^{\infty} \mathscr{D}\left(\pi\left(x_{0}\right)^{* n}\right)$.

Since $\pi\left(x_{0}\right)^{*}=\overline{\pi^{*}\left(x_{0}\right)}=\overline{\pi\left(x_{0}\right)}$, we have

$$
\mathscr{D}\left(\pi^{*}\right)=\bigcap_{n=1}^{\infty} \mathscr{D}\left({\overline{\pi\left(x_{0}\right)}}^{n}\right)=\bigcap_{n=1}^{\infty} \mathscr{D}\left({\overline{\pi^{*}\left(x_{0}\right)}}^{n}\right) .
$$

It follows from Theorem 2.1 that $\pi^{*}$ is selfadjoint.

We next give a suggestive example.

EXAMPLE 2.5. We deal with $L^{2}[0,1]$ as the fixed Hilbert space. Let $S$ be a closed operator defined by

$$
\begin{aligned}
\mathscr{D}(S) & =\left\{f \in C[0,1] ; f(x)-f(0)=\int_{0}^{x} g(t) d t \text { for some } g \in L^{2}[0,1]\right\}, \\
S f & =-i g \quad \text { for } f \in \mathscr{D}(S) \quad\left(f(x)-f(0)=\int_{0}^{x} g(t) d t\right) .
\end{aligned}
$$


We introduce closed operators obtained by $S$ as follows:

$$
\begin{aligned}
\mathscr{D}(T) & =\{f \in \mathscr{D}(S) ; f(1)=f(0)=0\}, \\
\mathscr{D}\left(T_{c}\right) & =\{f \in \mathscr{D}(S) ; f(1)=c f(0)\} \quad \text { for } c \in \mathbf{C}, \\
\mathscr{D}\left(T_{\infty}\right) & =\{f \in \mathscr{D}(S) ; f(0)=0\}, \\
T & =S \mid \mathscr{D}(T), \\
T_{c} & =S \mid \mathscr{D}\left(T_{c}\right) \text { for } c \in \mathbf{C} \cup\{\infty\} .
\end{aligned}
$$

Among these operators the following relations hold: $T^{*}=S, T_{c}^{*}=T_{c^{-1}}(c \neq 0)$, $T_{0}^{*}=T_{\infty}$ and $T \subset T_{c} \subset T^{*}$. These show that $T_{c}$ is selfadjoint if and only if $|c|=1$.

Let $Q$ be the free commutative algebra generated by an hermitian element $x_{0}$. We now define representations of $\Theta$ on $L^{2}[0,1]$ by:

$$
\begin{aligned}
& \mathscr{D}(\pi)=\bigcap_{n=1}^{\infty} \mathscr{D}\left(T^{n}\right)=\left\{f \in C^{\infty}[0,1] ; f^{(n)}(1)=f^{(n)}(0)=0, n=0,1,2, \ldots\right\}, \\
& \mathscr{D}\left(\pi^{c}\right)=\bigcap_{n=1}^{\infty} \mathscr{D}\left(T_{c}^{n}\right)=\left\{f \in C^{\infty}[0,1] ; f^{(n)}(1)=c f^{(n)}(0), n=0,1,2, \ldots\right\} \\
& \mathscr{D}\left(\pi_{\infty}\right)=\bigcap_{n=1}^{\infty} \mathscr{D}\left(T_{\infty}^{n}\right)=\left\{f \in C^{\infty}[0,1] ; f^{(n)}(0)=0, n=0,1, \ldots\right\}, \\
& \pi\left(x_{0}\right)=-i \frac{d}{d t} / \mathcal{D}(\pi), \quad \pi_{c}\left(x_{0}\right)=-i \frac{d}{d t} / \mathcal{D}\left(\pi_{c}\right) \quad(c \in \mathbf{C} \cup\{\infty\}) .
\end{aligned}
$$

Then we have the following results:

$$
\begin{gathered}
\text { Ol } \left.)\left(\pi^{*}\right)=C^{\infty}[0,1], \quad \pi^{*}\left(x_{0}\right)=-i \frac{d}{d t} / \oplus \backslash\right)\left(\pi^{*}\right) . \\
\pi \subset \pi_{c} \subset \pi^{*} \quad \text { for } c \in \mathbf{C} \cup\{\infty\} . \\
\pi^{* *}=\pi, \quad \pi_{c}^{*}=\pi_{c^{-1}} \quad(c \neq 0), \quad \pi_{0}^{*}=\pi_{\infty}, \quad \pi_{\infty}^{*}=\pi_{0} . \\
\pi_{c} \text { is a }{ }^{*} \text {-representation if and only if }|c|=1 .
\end{gathered}
$$

In this case, $\pi_{c}$ is selfadjoint.

$$
\begin{gathered}
\overline{\pi\left(x_{0}\right)}=T, \quad \overline{\pi^{*}\left(x_{0}\right)}=T^{*}, \quad \overline{\pi_{c}\left(x_{0}\right)}=T_{c} \quad(c \in \mathrm{C} \cup\{\infty\}) . \\
\mathscr{D}(\pi)=\bigcap_{n=1}^{\infty} \mathscr{D}\left({\overline{\pi\left(x_{0}\right)}}^{n}\right), \quad \mathscr{D}\left(\pi^{*}\right)=\bigcap_{n=1}^{\infty} \mathscr{D}\left({\overline{\pi^{*}\left(x_{0}\right)}}^{n}\right), \\
\mathscr{D}\left(\pi_{c}\right)=\bigcap_{n=1}^{\infty} \mathscr{D}\left({\overline{\pi_{c}\left(x_{0}\right)}}^{n}\right) \quad \text { for } c \in \mathrm{C} \cup\{\infty\} .
\end{gathered}
$$

We next define subrepresentations of $\pi_{c}(c \in \mathbf{C} \cup\{\infty\})$ by:

$$
\begin{gathered}
\mathscr{D}\left({ }_{n} \pi_{c}\right)=\left\{f \in \mathscr{D}\left(\pi_{c}\right) ; f^{(k)}(1)=f^{(k)}(0)=0, k=n, n+1, \ldots\right\}, \\
{ }_{n} \pi_{c}\left(x_{0}\right)=\pi_{c}\left(x_{0}\right) / \mathscr{D}\left({ }_{n} \pi_{c}\right) \text { for } n=1,2, \ldots .
\end{gathered}
$$

Then we have the following results:

$$
\pi \varlimsup_{1} \pi_{c} \subsetneq \cdots \subsetneq_{n} \pi_{c} \subsetneq \cdots \subsetneq \pi_{c} .
$$




$$
\begin{gathered}
{ }_{n} \pi_{c}^{*}=\pi_{c}^{*} \quad \text { for } n=1,2, \ldots \\
{ }_{n} \pi_{c} \text { is a *-representation if and only if }|c|=1 . \\
\overline{{ }_{n} \pi_{c}\left(x_{0}\right)}=T_{c} \quad \text { for } n=1,2, \ldots . \\
\mathscr{D}\left({ }_{n} \pi_{c}\right) \underset{F}{\subsetneq} \bigcap_{k=1}^{\infty} \mathscr{D}\left({\overline{{ }_{n} \pi_{c}\left(x_{0}\right)}}^{k}\right)=\mathscr{D}\left(\pi_{c}\right) .
\end{gathered}
$$

Remark. Let $Q$ be the commutative algebra generated by an hermitian element $x_{0}$ and $\pi$ a closed ${ }^{*}$-representation of $\mathbb{Q}$ on a Hilbert space.

(1) Although $\pi^{*}$ is selfadjoint if $\pi\left(x_{0}\right)$ is essentially selfadjoint, $\pi$ is not always selfadjoint (see Example 2.5 (7), (9), (10)).

(2) The condition $\mathscr{D}=\bigcap_{n=1}^{\infty} \mathscr{D}\left(\overline{\pi\left(x_{0}\right)^{n}}\right)$ does not always imply that $\pi\left(x_{0}\right)$ is essentially selfadjoint (see Example 2.5(1), (6) and Theorem 2.1).

(3) The essentially selfadjointness of $\pi\left(x_{0}\right)$ does not always imply the condition Q $=\cap_{n=1}^{\infty} \mathcal{D}\left(\overline{\pi\left(x_{0}\right)^{n}}\right)$ (see (1) and Theorem 2.1).

(4) The essentially selfadjointness of $\pi\left(x_{0}\right)$ does not always imply that of $\pi\left(x_{0}\right)^{n}$ for $n=1,2, \ldots$ (see Example 2.5(7), (10) and Theorem 2.1).

(5) Let $\pi$ be a closed ${ }^{*}$-representation of a ${ }^{*}$-algebra $Q$. Although $\pi(Q)^{\prime}$ is a von Neumann algebra if the condition $\pi(Q)^{\prime} \mathscr{Q}(\pi)=\mathscr{Q}(\pi)$ holds, the converse does not always hold (see Example 2.5(7), (8), (9), Theorem 2.1 and Lemma 2.3).

3. Standardness of $\mathscr{P}(A, B)$. In $\S 2$ we have shown that $(\mathscr{P}(A)$, $\mathscr{D})$ is selfadjoint if and only if $(\mathscr{P}(A), \mathscr{D})$ is standard. But in the $O_{p}^{*}$-algebra $(\mathcal{P}(A, B), \mathscr{D})$ generated by two commuting hermitian operators $A$ and $B$, selfadjointness does not always imply standardness [8, Example $3, \S \mathrm{V}]$. In this section we investigate the standardness of $\mathscr{P}(A, B)$.

THEOREM 3.1. Let $\mathcal{P}(x, y)$ be the free commutative algebra on two hermitian generators $x$ and $y$. Let $\pi_{0}$ be $a^{*}$-representation of $\mathcal{P}(x, y)$ on a Hilbert space $\mathfrak{h}$. Then the following statements are equivalent.

(1) There exists a standard representation $\pi$ of $\mathscr{P}(x, y)$ on $\mathfrak{h}$ which is an extension of $\pi_{0}$.

(2) There exists a normal operator $C$ on $\mathfrak{h}$ which is an extension of $\pi_{0}(x+i y)$.

In this case, we can define $\pi$ as follows:

$$
\mathscr{D}(\pi)=\bigcap_{n=1}^{\infty} \mathscr{D}\left(C^{n}\right)=\bigcap_{n=1}^{\infty}\left(\mathscr{D}\left(A^{n}\right) \cap \mathscr{D}\left(B^{n}\right)\right)
$$

and

$$
\pi(x)=A|\mathscr{D}(\pi), \quad \pi(y)=B| \mathscr{D}(\pi),
$$

where $A=\frac{1}{2} \overline{\left(C+C^{*}\right)}$ and $B=\frac{1}{2 i} \overline{\left(C-C^{*}\right)}$.

Proof. $(1) \Rightarrow(2)$ : Let $\pi$ be a standard representation stated above. Then $\overline{\pi(x+i y)}$ is a normal operator satisfying the condition mentioned in (2).

$(2) \Rightarrow(1)$ : Let $C$ be a normal operator on $\mathfrak{h}$ which is an extension of $\pi_{0}(x+i y)$. We put $A=\frac{1}{2} \overline{\left(C+C^{*}\right)}$ and $B=\frac{1}{2 i} \overline{\left(C-C^{*}\right)}$. Then $A$ and $B$ are selfadjoint operators with mutually commuting spectral projections. Let $A=\int \lambda d E_{A}(\lambda)$ and 
$B=\int \lambda d E_{B}(\lambda)$ be the spectral resolutions of $A$ and $B$. We put $E(\lambda, \mu)=$ $E_{A}(\lambda) E_{B}(\mu)$. Then for each polynomial $P(\lambda, \mu)$ of two variables, we can define a closed operator $P(A, B)$ on $\mathfrak{h}$ as follows:

$$
\begin{aligned}
\mathscr{D}(P(A, B)) & =\left\{\xi \in \mathfrak{h} ; \int|P(\lambda, \mu)|^{2} d\|E(\lambda, \mu) \xi\|^{2}<\infty\right\}, \\
P(A, B) \xi & =\int P(\lambda, \mu) d E(\lambda, \mu) \xi \text { for } \xi \in \mathscr{D}(P(A, B)) .
\end{aligned}
$$

In particular, putting $P(\lambda, \mu)=\lambda+i \mu$, we get $C=P(A, B)$. When we put $\bar{P}(\lambda, \mu)$ $=\overline{P(\lambda, \mu)}$ for any real $\lambda, \mu$, it is known that $P(A, B)^{*}=\bar{P}(A, B)$. When we put $\mathscr{D}(\pi)=\bigcap_{n=1}^{\infty} \mathcal{D}\left(C^{n}\right)$, it is clear that

$$
\mathscr{D}\left(\pi_{0}\right) \subset \mathscr{D}(\pi) \text { and } \mathscr{D}(\pi)=\bigcap_{p(\lambda, \mu)} \mathscr{D}(P(A, B))=\bigcap_{n=1}^{\infty}\left(\mathscr{D}\left(A^{n}\right) \cap \mathscr{D}\left(B^{n}\right)\right) \text {, }
$$

for we have $\mathscr{D}\left(C^{n}\right)=\mathscr{D}\left(A^{n}\right) \cap \mathscr{Q}\left(B^{n}\right)$. We define an $O_{p}^{*}$-algebra $\mathscr{P}\left(A_{0}, B_{0}\right)$ on $\mathcal{Q}(\pi)$ as follows:

$$
\begin{gathered}
A_{0}=A\left|\mathscr{D}(\pi), \quad B_{0}=B\right| \mathcal{D}(\pi), \quad P\left(A_{0}, B_{0}\right)=P(A, B) \mid \mathscr{D}(\pi), \\
\mathscr{P}\left(A_{0}, B_{0}\right)=\left\{P\left(A_{0}, B_{0}\right) \mid P(\lambda, \mu) \text { : polynomial }\right\} .
\end{gathered}
$$

The involution ${ }^{+}$on $\mathscr{P}\left(A_{0}, B_{0}\right)$ is defined by $P\left(A_{0}, B_{0}\right)^{+}=\bar{P}\left(A_{0}, B_{0}\right)$. It is clear that $\left(\mathscr{P}\left(A_{0}, B_{0}\right), \mathscr{Q D}(\pi)\right)$ is closed. Now we show that $\left(\mathscr{P}\left(A_{0}, B_{0}\right), \mathscr{D}(\pi)\right)$ is standard. We put

$$
E_{n}=\left(E_{A}(n)-E_{A}(-n)\right)\left(E_{B}(n)-E_{B}(-n)\right)
$$

for any natural number $n$. Let $P(\lambda, \mu)$ be a polynomial and $\xi$ an element of

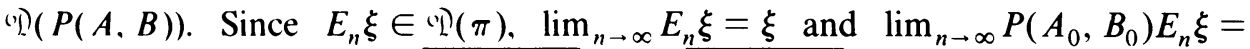
$P(A, B) \xi$. we have $\xi \in(1)\left(\overline{P\left(A_{0}, B_{0}\right)}\right)$ and $\overline{P\left(A_{0}, B_{0}\right)} \xi=P(A, B) \xi$. This means that $\overline{P\left(A_{0}, B_{0}\right)}=P(A, B)$. Consequently,

$$
\begin{aligned}
P\left(A_{0}, B_{0}\right)^{*} & =\overline{P\left(A_{0}, B_{0}\right)} *=P(A, B)^{*}=\bar{P}(A, B) \\
& =\overline{P\left(A_{0}, B_{0}\right)}=\overline{P\left(A_{0}, B_{0}\right)^{+}} .
\end{aligned}
$$

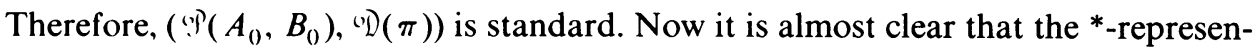
tation $\pi$ of $\varphi P(x, y)$ on $\mathfrak{h}$ defined by $\pi(P(x, y))=P\left(A_{0}, B_{0}\right)$ satisfies the condition mentioned in (1).

TheOREM 3.2. Let $\mathscr{D}$ be a dense subspace of a Hilbert space $\mathfrak{h}$. Let $A$ and $B$ be hermitian elements of $\mathcal{L}^{+}(\mathcal{Q})$ satisfying $A B=B A$ and $\mathscr{P}(A, B)$ the commutative $O_{p}^{*}$-algebra on $\mathscr{D}$ generated by $A$ and $B$. Then the following statements are equivalent.

(1) $(\mathscr{P}(A, B), \mathscr{D})$ is standard.

(2) $(\mathscr{P}(A, B), \mathscr{D})$ is selfadjoint and there exists a normal operator $C$ which is an extension of $A+i B$.

(3) $A$ and $B$ are essentially selfadjoint, $\mathscr{D}=\bigcap_{n=1}^{\infty}\left(\mathscr{D}\left(\bar{A}^{n}\right) \cap \mathscr{D}\left(\bar{B}^{n}\right)\right)$ and there exists a normal operator $C$ which is an extension of $A+i B$.

(4) $\bar{A}$ and $\bar{B}$ are selfadjoint with mutually commuting spectral projections and $(\mathscr{P}(A, B), \mathscr{D})$ is a closed $O_{p}^{*}$-algebra satisfying $\mathscr{P}(A, B)^{\prime} \mathscr{D}=\mathscr{\bigotimes}$. 
Proof. (1) $\Rightarrow(2)$ : Suppose that $(\mathscr{P}(A, B), \mathscr{Q})$ is standard. Then $(\mathscr{P}(A, B), \mathscr{D})$ is selfadjoint and $\overline{A+i B}$ is normal.

$(2) \Rightarrow(3)$ : Let $\pi_{0}$ be the identity representation of $\mathcal{P}(A, B)$ on $\mathfrak{h}$. Then by the assumption and Theorem 3.1, there exists a standard representation $\pi$ of $\mathcal{P}(A, B)$ on $\mathfrak{h}$, which is an extension of $\pi_{0}$, with $\mathscr{D}(\pi)=\bigcap_{n=1}^{\infty}\left(\mathscr{D}\left(A_{1}^{n}\right) \cap \mathscr{D}\left(B_{1}^{n}\right)\right), \pi(A)=$ $A_{1} \mid \mathscr{D}(\pi)$ and $\pi(B)=B_{1} \mid \mathscr{D}(\pi)$, where $A_{1}=\frac{1}{2} \overline{\left(C+C^{*}\right)}$ and $B_{1}=\frac{1}{2 i} \overline{\left(C-C^{*}\right)}$. On the other hand, the selfadjointness of $\pi_{0}$ shows that $\pi_{0}$ is identical to $\pi$, for the selfadjoint representation of a ${ }^{*}$-algebra is maximal. Therefore $A$ and $B$ are essentially selfadjoint. Furthermore, $A \subset A_{1}$ (resp. $B \subset B_{1}$ ) and the selfadjointness of $A_{1}$ (resp. $B_{1}$ ) shows that $\bar{A}=A_{1}$ (resp. $\bar{B}=B_{1}$ ). Therefore,

$$
\mathscr{D}=\mathscr{D}(\pi)=\bigcap_{n=1}^{\infty}\left(\mathscr{D}\left(\overline{A^{n}}\right) \cap \mathscr{D}\left(\bar{B}^{n}\right)\right) \text {. }
$$

$(3) \Rightarrow(4)$ : As in the proof of $(2) \Rightarrow(3)$ we see that $\bar{A}$ and $\bar{B}$ are selfadjoint operators with mutually commuting spectral projections. The selfadjointness of $\mathscr{P}(A, B)$ implies that $\mathcal{P}(A, B)$ is closed and $\mathcal{P}(A, B)^{\prime} \mathscr{D}=\mathscr{Q}$.

(4) $\Rightarrow(1)$ : Since the spectral projections $E_{A}(\lambda)$ and $E_{B}(\mu)$ of the selfadjoint operators $\bar{A}$ and $\bar{B}$ are mutually commuting, we have $E_{A}(\lambda), E_{B}(\mu) \in \mathscr{P}(A, B)^{\prime}=$ $\mathcal{P}(A)^{\prime} \cap \mathcal{P}(B)^{\prime}$ for $-\infty<\lambda, \mu<\infty$. Hence, $\mathscr{P}(A, B)^{\prime \prime} \subset \mathcal{P}(A, B)^{\prime}$. Furthermore, since $\mathscr{P}(A, B)^{\prime} \mathscr{D}=\mathscr{D}$, it follows that $\mathscr{P}(A, B)^{\prime \prime} \mathscr{D}=\mathscr{Q}$ and $\overline{P(A, B)}$ is affiliated with the von Neumann algebra $\mathcal{P}(A, B)^{\prime \prime}$ for each $P(A, B) \in \mathcal{P}(A, B)$. Then the $O_{p}^{*}$-algebra $(\mathfrak{A}, \mathscr{D})$ generated by $\mathscr{P}(A, B)$ and $\mathscr{P}(A, B)^{\prime \prime}$ becomes a closed $E W^{\#}$. algebra on $\mathscr{D}$ over $\mathscr{P}(A, B)^{\prime \prime}$, so that $\mathfrak{A}$ is standard by [5, Theorem 2.3]. Hence, $\mathcal{P}(A, B)$ is standard.

REMARK. We note that we can prove a theorem similar to Theorems 3.1 and 3.2 for the *-algebra $\mathscr{P}\left(x_{1}, \ldots, x_{n}\right)$ generated by $n$ commuting hermitian elements $x_{1}, \ldots, x_{n}$.

\section{REFERENCES}

1. H. J. Borchers, Algebraic aspects of Wightman field theory, Statistical Mechanics and Field Theory Lectures (Haifa Summer School, 1971), New York, Jerusalem and London, 1972, pp. 31-79.

2. J. Dixmier, Les algèbres d'opérateurs dans l'espace Hilhertian, 2ième ed., Gauthier-Villars, Paris, 1969.

3. N. Dunford and J. T. Schwartz, Linear operators. II, Interscience, New York, 1957.

4. S. P. Gudder and W. Scruggs, Unbounded representations of *algebras, Pacific J. Math. 70 (1977), $369-382$.

5. A. Inoue, On a class of unbounded operator algebras, Pacific J. Math. 65 (1976), 77-95.

6. G. Lassner, Topological algebras of operators, Rep. Math. Phys. 3 (1972), 279-293.

7. E. Nelson, Analytic vectors, Ann. of Math. (2) 70 (1959), 572-615.

8. R. T. Powers, Self-adjoint algebras of unbounded operators, Comm. Math. Phys. 21 (1971), 85-124.

9. R. T. Powers, Self-adjoint algebras of unbounded operators. II, Trans. Amer. Math. Soc. 187 (1974), 261-293.

10. K. Schmüdgen, The order structure of topological *-algebras of unbounded operators, Rep. Math. Phys. 7 (1975), 215-227.

11. K. Takesue, Spatial theory for algebras of unbounded operators, Rep. Math. Phys. (to appear).

Department of ApPlied Mathematics, Fukuoka University, 814-01 FukUOKa, Japan 